\title{
Modélisation numérique et expérimentale du comportement de sous-ensembles de ballons pressurisés stratosphériques
}

\author{
Pierre Vialettes $^{1, a}$, Jean-Michel Siguier ${ }^{1}$, Olivier Dalverny ${ }^{2}$, Sébastien Mistou ${ }^{2}$, \\ Moussa Karama ${ }^{2}$ et Frank Petitjean ${ }^{3}$ \\ 1 ONERA Centre de Toulouse BP 40252, avenue Édouard Belin, 31055 Toulouse Cedex 4, France \\ 2 Laboratoire Génie de Production, École Nationale d'Ingénieurs de Tarbes, BP 1629, 65016 Tarbes Cedex, France \\ 3 Institut Catholique des Arts et Métiers, Avenue de Grande Bretagne, 31000 Toulouse, France
}

Reçu le 25 février 2005, accepté le 21 mars 2005

\begin{abstract}
Résumé - Ce travail concerne la simulation numérique et expérimentale du comportement mécanique des ballons pressurisés stratosphériques. Ce programme de recherche entrepris par le CNES et l'ONERA est basé sur (i) la modélisation du matériau polymère constitutif des ballons par des lois de comportement de type viscoélastique; (ii) la simulation expérimentale de sous-ensembles de ballons lors de phases de vol; (iii) la validation des lois viscoélastiques par comparaison essais/calculs du comportement de sous-ensembles de ballons. Le dispositif expérimental utilisé, nommé NIRVANA, reproduit les conditions de vol d'un ballon, i.e. la température, la pression et la contrainte dans l'enveloppe. Dans le cadre des caractérisations en grandes déformations des polymères, l'instrumentation généralement mise en œuvre permet seulement d'accéder à des caractéristiques globales et macroscopiques. Le suivi de tels essais par la méthode de stéréo-corrélation d'images, méthode basée sur les principes de la photogrammétrie, permet d'accéder de manière fine aux champs de déplacements et de déformations sur la totalité de la surface mesurée. Cette richesse d'information expérimentale s'avère particulièrement utile pour la validation non seulement globale mais aussi locale des lois de comportement utilisées.
\end{abstract}

Mots clés : Mesure sans contact / stéréo-corrélation / polymères / ballons stratosphériques / viscoélasticité / identification

\begin{abstract}
Numerical and experimental modeling of the behavior of pressurized stratospheric balloons subsets. This work relates to numerical simulation and experimental mechanical behavior of the stratospheric pressurized balloons. This research program carried out by the CNES and ONERA is based on (i) the modeling of polymeric material constitutive of the balloons behavior laws of viscoelastic type; (ii) the experimental simulation of balloons subsets in flight phases; (iii) validation of the viscoelastic laws by comparison tests/calculations of the balloons subsets behavior. The facility named NIRVANA reproduces the flying conditions of a balloon, i.e. the temperature, the pression and the constraint in the balloon envelope. In the context of identification of polymer large deformation behaviour, general measurement only brings to global and macroscopic characteristics. The follow-up of these tests by stereo correlation of images, method based on photogrammetry principles, gives access to displacements and strain fields with accuracy on the totally measured area. This wealth of experimental information is particularly useful for the global and local verification of the behavior laws.
\end{abstract}

Key words: Non-contact measurement / stereo-correlation / polymer / stratospheric balloons / viscoelasticity / identification

${ }^{a}$ Auteur correspondant : vialette@onecert.fr 


\section{Nomenclature}

\begin{tabular}{|c|c|}
\hline$x, y$ & coordonnées de l'image \\
\hline$x_{0}, y_{0}$ & coordonnées du point principal \\
\hline$d_{x}, d_{y}$ & distorsions des objectifs \\
\hline$c$ & constante représentative des caméras \\
\hline$R$ & matrice de rotation \\
\hline$X_{0}, Y_{0}, Z_{0}$ & coordonnées du centre de projection \\
\hline$X, Y, Z$ & coordonnées du point objet \\
\hline$\sigma$ & précontrainte \\
\hline$\Delta P$ & différence de pression entre l'intérieur et l'extérieur du ballon \\
\hline$R$ & rayon du ballon \\
\hline$e$ & épaisseur de l'enveloppe \\
\hline$t_{0}$ et $t_{\infty}$ & temps de début et d'équilibre en fluage \\
\hline$\xi_{i}$ & écoulement visqueux \\
\hline$\tau_{i}$ & temps de relaxation \\
\hline$\mu_{i}$ & coefficient de pondération (gaussienne) \\
\hline$n_{0}, n_{\mathrm{c}}$ & spectre gaussien \\
\hline$i \in\left[n_{1}, n_{2}\right]$ & caractéristiques du spectre de la gaussienne \\
\hline $\bar{\sigma}$ & contrainte équivalente \\
\hline $\bar{\varepsilon}$ & déformation équivalente \\
\hline$T$ & température \\
\hline$A, q, c, m$ & coefficients \\
\hline$t$ & temps \\
\hline$L$ & tenseur d'ordre 4 d'anisotropie élastique \\
\hline$\varepsilon$ & déformation totale \\
\hline$\varepsilon^{a}$ & déformation anélastique \\
\hline$g(\sigma)$ & fonction non linéarisante \\
\hline$\beta$ & paramètre \\
\hline$L_{R}$ & tenseur d'ordre 4 d'anisotropie visqueux \\
\hline$\gamma_{R}$ & coefficient lié à la viscosité \\
\hline$v_{R}$ & coefficient de Poisson du tenseur relaxé \\
\hline$\gamma_{\mathrm{c}}$ & coefficient représentant la viscosité en cisaillement \\
\hline$v$ & coefficient de Poisson \\
\hline$S_{R}=L_{R}^{-1}$ & tenseur de souplesse \\
\hline
\end{tabular}

\section{Introduction}

Les ballons pressurisés (Fig. 1a) sont développés par le CNES pour effectuer des expériences de longue durée dans la stratosphère. Ils sont dimensionnés en considérant que les matériaux ont un comportement linéaire jusqu'à leur seuil d'élasticité [1-3]. Durant la phase de développement, deux problèmes principaux ont été mis en évidence et doivent être résolus.

Le premier de ces problèmes est l'éclatement prématuré de certains ballons en vol apparaissant à des niveaux de pression relativement faibles. Le second est apparu lors du dépouillement des résultats d'essais en vol. Ces ballons ont un diamètre initial de $10 \mathrm{~m}$, avec un poids bien connu et donc un niveau de vol précisément calculé. Il s'avère que le fluage du polymère constitutif de ces ballons entraîne une augmentation significative du diamètre et modifie donc tous les paramètres du vol.

Les ballons pressurisés de $10 \mathrm{~m}$ de diamètre sont composés de fuseaux assemblés par un ruban de Polyester
(PET) et de colle thermo-réactivable (Fig. 1b). Les fuseaux se rejoignent au niveau des pôles et sont repris par une calotte en film PET de différentes épaisseurs. Le matériau constitutif des fuseaux est un film complexé tri-couches composé de Polyester $15 \mu \mathrm{m}$, de Polyamide $20 \mu \mathrm{m}$ et de Polyester $15 \mu \mathrm{m}$ (PET/PA/PET), pour une épaisseur totale de $50 \mu \mathrm{m}$. L'objectif de cette étude est de modéliser le comportement non-linéaire de ces ballons pressurisés prenant en compte l'influence du temps (fluage, relaxation).

On identifie les lois de comportement du matériau à partir d'essais uniaxiaux en traction, fluage et relaxation. Ces essais se font sur une machine de traction équipée d'une camera CCD (Charge Coupled Device) permettant la mesure du champ de déplacement et le calcul des déformations dans le plan de sollicitation de l'éprouvette [4-7]. Cette méthode non intrusive est adaptée à l'identification du comportement mécanique de matériaux souples et transparents. La mesure du champ de déplacement nécessite le dépôt sur l'éprouvette d'un 

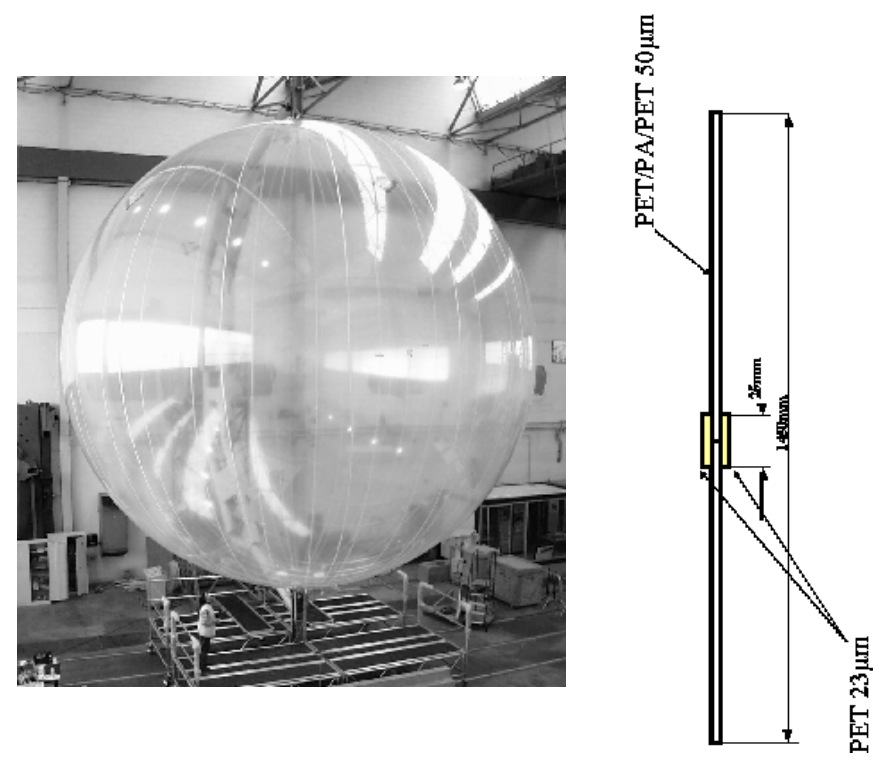

Fig. 1. Ballon pressurisé $10 \mathrm{~m}$ (a) et assemblage de fuseaux (b).

mouchetis. Le polymère utilisé étant sensible aux solvants des peintures, une étude a été menée afin de valider l'utilisation de tels dépôts [8-10]. L'aspect viscoélastique de ce polymère le rend sensible aux variations de vitesses de sollicitations. Une méthode a été mise au point afin de pouvoir prendre un nombre d'images significatif, notamment dans la zone élastique, à des vitesses de sollicitations de l'ordre de $100 \mathrm{~mm} . \mathrm{min}^{-1}$. Ces essais ont été réalisés de la température ambiante jusqu'à $-60{ }^{\circ} \mathrm{C}$. Une procédure expérimentale spécifique est nécessaire pour les mesures à froid. Cette procédure est appliquée pour mesurer le coefficient de dilatation du matériau [8].

La validation des lois identifiées [11-13] se fait à partir d'essais représentatifs des phases de vol sur le dispositif expérimental NIRVANA. Ce moyen d'essai permet de mesurer via un système de stéréo-corrélation d'images les déplacements et de déduire les déformations d'un sous-ensemble de ballon soumis à des conditions de température et de pression représentatives des phases de vol. Des essais ont été menés à température ambiante et jusqu'à $-60{ }^{\circ} \mathrm{C}$ sur des calottes et des assemblages sous des sollicitations de traction à vitesse de mise en pression constante ainsi qu'à pression constante durant plusieurs jours $[14,15]$. Le matériau étudié étant un réflecteur spéculaire semi-transparent, les échantillons sont préparés afin de pouvoir mesurer les déplacements.

\section{Identification du comportement du matériau}

\subsection{Matériaux étudiés}

Les ballons pressurisés stratosphériques de $10 \mathrm{~m}$ de diamètre sont constitués de fuseaux en film multicouche de $50 \mu \mathrm{m}$ d'épaisseur, assemblés par collage de bandes de
PET de $23 \mu \mathrm{m}$ d'épaisseur (Fig. 1b). Le film complexe est un polymère tri-couches obtenu à partir de 2 films de PET de $15 \mu \mathrm{m}$ d'épaisseur entre lesquels s'intercale un film de polyamide (PA) de $20 \mu \mathrm{m}$ d'épaisseur (PET/PA/PET). Les principales propriétés de ce complexe sont sa légèreté, sa bonne résistance mécanique alliée à une bonne souplesse jusqu'à de très basses températures, son étanchéité aux gaz aérostatiques et sa résistance aux UV et à l'ozone $[2,15,16]$. Hormis le gain en souplesse, l'intérêt du complexage de 3 films est d'éviter les fuites dues aux éventuels micro-trous pouvant apparaître sur chaque film [10]. Enfin, il offre une bonne résistance bi-axiale avec un coefficient d'isotropie supérieur à 0,9 , malgré le procédé de mise en forme des 3 films, par bi-étirage.

\subsection{Identification des caractéristiques thermoélastiques}

La principale difficulté de l'identification des caractéristiques mécaniques de matériaux souples et transparents comme le complexe PET/PA/PET est la connaissance du coefficient de Poisson. Les méthodes trop intrusives telles que les jauges de déformation influent sur le comportement global du polymère. Pour déterminer les déformations dans le plan de sollicitation de l'éprouvette lors d'un essai de traction uniaxial, on peut mettre en œuvre des procédés d'extensométrie sans contact tels que la corrélation d'images.

\subsubsection{Mesures sans contact par stéréo-corrélation d'images}

Les deux caméras CCD permettent d'acquérir des images numériques avec une définition de 1280 par 1024 pixels. Ces images sont ensuite traitées avec un logiciel spécifique de reconnaissance d'images. Grâce à ce dispositif il est possible de mesurer les déplacements et les déformations à la surface d'un objet avec une résolution de 1000 à $2000 \mu \mathrm{m} . \mathrm{m}^{-1}$ en déformations. Ce dispositif est donc adapté à des comportements de grandes déformations [1-4].

Le logiciel est basé sur le principe de photogrammétrie qui est une des méthodes de mesure optique qui permet de mesurer les coordonnées 3D de points sur la surface d'un objet. À partir de ces coordonnées, il est possible de calculer le vecteur de déplacement, les déformations locales et les différences de formes si l'objet se déforme [4-6].

Si la position de 2 caméras et de 2 points images homologues $p_{1}\left(x_{1}, y_{1}\right)$ et $p_{2}\left(x_{2}, y_{2}\right)$ sont connus, le point objet correspondant $P(X, Y, Z)$ peut être calculé (Fig. 2). Cette procédure de calcul est connue sous le nom d'intersection spatiale.

Pour cela, un modèle géométrique doit être défini, qui décrit la transformation permettant de passer des points image au point physique. On peut alors utiliser le modèle de projection par perspective qui est très souvent utilisé 


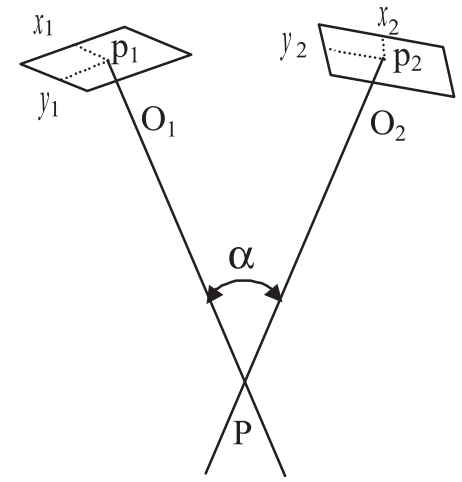

Fig. 2. Méthode de l'intersection spatiale.

en photogrammétrie. Son modèle mathématique repose sur la transformation colinéaire $[4,5,12]$

$$
\left\{\begin{array}{c}
x-x_{0}-d_{x} \\
y-y_{0}-d_{y} \\
c
\end{array}\right\}=R\left\{\begin{array}{c}
X-X_{0} \\
Y-Y_{0} \\
Z-Z_{0}
\end{array}\right\}
$$

$x, y$ sont les coordonnées de l'image, $x_{0}, y_{0}$ les coordonnées du point principal, $d_{x}, d_{y}$ les distorsions des objectifs, $c$ une constante représentative des caméras, $R$ la matrice de rotation, $X_{0}, Y_{0}, Z_{0}$ les coordonnées du centre de projection, $X, Y, Z$ les coordonnées du point objet.

Les paramètres de la matrice de rotation (décrite par les angles d'Euler $\omega, \phi, k)$ et les paramètres du centre de projection $\left(X_{0}, Y_{0}, Z_{0}\right)$ sont connus comme « orientation extérieure $»$, les paramètres $x_{0}, y_{0}, d_{x}, d_{y}$ et $c$ sont connus comme « orientation intérieure » des caméras. Ils doivent être calculés lors d'une phase de calibration.

La calibration est l'étape fondamentale qui précède l'acquisition d'images. Elle sert à déterminer tous les paramètres géométriques nécessaires au calcul de la transformation colinéaire : la distance et l'angle entre les caméras, la distance foyer/objectif. Elle permet de quantifier les aberrations optiques afin qu'elles puissent être prises en compte dans les calculs. On utilise une plaque de calibration de dimensions équivalentes à la surface de mesure, que l'on place à une distance de mesure fixe. Au cours de ce processus, plusieurs images de la plaque sont prises dans différentes positions. La plaque de calibration est constellée de points blancs dont l'éloignement est connu. La résolution obtenue au cours de la calibration est inférieure au pixel, ce qui correspond à une erreur de quelques micromètres en déplacements.

Le logiciel de traitement d'images est capable de reconnaître les points d'une image par analyse des niveaux de gris. Cela est rendu possible grâce à l'application sur l'objet à mesurer de peintures blanche et noire qui forment des niveaux de gris variables sur la surface de l'objet. À chaque pixel des capteurs CCD correspond une valeur de niveau de gris codée sur 8 ou 12 bits. Une suite de plusieurs pixels pris sur une même ligne forme une séquence de niveaux de gris appelée distribution de niveaux de gris. Le principe fondamental réside dans le fait que les distributions ne varient pas au cours de la déformation de l'objet. Il suffit donc de suivre ces distributions de niveau de gris dans leur mouvement pour avoir les déplacements de l'objet. En pratique, cela est rendu possible grâce à des jauges optiques qui sont des bases de mesure regroupant $n \times n$ pixels.

Le centre de chaque jauge optique est repéré à partir de la distribution des niveaux de gris des pixels, situés sur deux droites orthogonales passant par le centre de la jauge. Quatre centres de jauges optiques constituent les sommets d'une maille. L'ensemble de toutes les mailles forme un maillage de la surface de l'objet. Lorsque le centre des jauges bouge au cours d'une déformation, les mailles se déforment, tournent et se translatent. Les pixels de la maille de l'image de référence sont alors transformés dans l'image de destination. Afin de compenser automatiquement d'éventuelles variations de luminosité au cours des mesures, une transformation linéaire de la luminosité est utilisée simultanément au processus de comparaison des images.

Pour comparer deux images, il est nécessaire de rechercher des distributions horizontales et verticales de niveaux de gris qui soient identiques. Il faut donner un point de départ dans les quatre images que l'on compare (images de référence et images après déformation des caméras de gauche et de droite). Ces quatre points sont des points homologues car ils correspondent au même point physique vu par les deux caméras, avant et après déformation.

\subsubsection{Essais mécaniques}

Ces essais ont été réalisés sur une machine de traction équipée d'un capteur d'effort de 1000 N. La vitesse de sollicitation choisie pour ces essais $\left(100 \mathrm{~mm} . \mathrm{min}^{-1}\right)$ permet de s'affranchir d'une grande partie des effets de fluage sur l'éprouvette.

La mesure par corrélation d'images nécessite un dépôt de peinture sur l'échantillon à tester. Les polymères étant sensibles aux solvants, une étude a été menée afin de choisir un dépôt adéquat. La peinture utilisée est en fait un primaire blanc. Ce produit, dans son utilisation d'origine s'applique en sous-couche afin de faire adhérer au mieux la première couche de peinture.

Une première série d'essais est effectuée sur un nombre d'éprouvettes suffisant pour obtenir une bonne reproductibilité. Une deuxième série d'essais est effectuée sur le même nombre d'éprouvettes peintes avec au minimum 30 min de séchage.

Ces essais se font sur le complexe PET/PA/PET en traction monotone à $100 \mathrm{~mm} \cdot \mathrm{min}^{-1}$ jusqu'à rupture. Les mesures comparatives effectuées sur le module d'Young tangent, la déformation et la charge à rupture ont montré que le primaire appliqué à ce matériau n'altère donc pas de façon sensible ses caractéristiques mécaniques principales [8].

Le matériau étant sensible à la vitesse de sollicitation [1], une vitesse de traction relativement élevée $\left(100 \mathrm{~mm} . \mathrm{min}^{-1}\right)$ permet de s'affranchir d'une grande partie des phénomènes de fluage. À cette vitesse, les caméras utilisées ne permettent de capturer qu'une seule image 


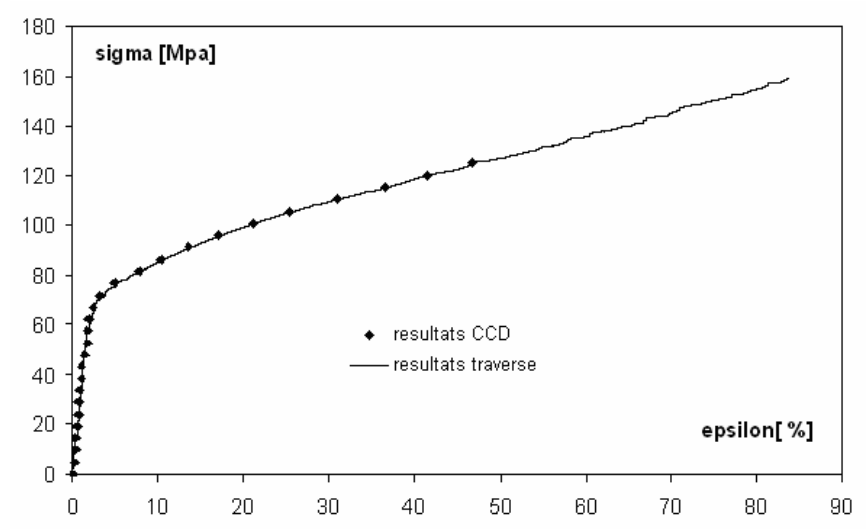

Fig. 3. Courbe conventionnelle du complexe PET/PA/PET.

par seconde et l'acquisition d'une image se fait par un signal extérieur issu de la machine de traction. Ce signal analogique est proportionnel à la charge. Nous avons donc choisi de prendre une image tous les $20 \mathrm{~N}$ afin d'obtenir environ 3 images dans la zone élastique, cette limite ayant été identifiée à $60 \mathrm{~N}$. Cette procédure permet de tracer la courbe conventionnelle de traction (Fig. 3).

Dans le cas où le résultat de l'essai attendu ne nécessite pas le recoupement avec la charge, comme, par exemple, la mesure du coefficient de Poisson ou seule la déformation est nécessaire, la capture d'image par un signal extérieur n'est pas nécessaire. Ainsi, pour mesurer le coefficient de Poisson (Fig. 5), nous utilisons une méthode de capture en fonction du temps qui permet de prendre jusqu'à 4 images par secondes. La différence de cadence de prise d'image avec la méthode précédente est due au fait que l'on ne perd pas de temps en lecture d'entrée analogique. La capture temporelle se fait avec l'horloge interne de l'ordinateur.

L'essai consiste donc à prendre une rafale d'images à partir d'un signal de départ donné par la machine de traction. Ce signal a été réglé afin que l'acquisition débute dès que la charge dans l'éprouvette dépasse 1,5 N. Ensuite, le système de mesure prend une image toutes les $0,25 \mathrm{~s}$. Le dépouillement des calculs permet, connaissant la limite élastique en déformation, de discriminer les images correspondant à la zone élastique et à la zone plastique.

\subsubsection{Mesure du coefficient de dilatation}

Le domaine de vol des ballons nécessite une bonne connaissance du comportement des matériaux jusqu'à $-60{ }^{\circ} \mathrm{C}$. L'objectif de l'essai (réalisé sur la machine de traction citée ci-dessus avec une enceinte thermique refroidie à l'azote et équipée d'un hublot) est de mesurer la déformation d'une éprouvette (PET/PA/PET) soumise à une variation de température. Seule la déformation due à la température doit être prise en compte. On effectue donc un essai de fluage à très faible charge (environ $0,5 \mathrm{~N}$ ) sur l'éprouvette à tester. Cette charge entraîne des déformations très faibles devant celles dues à la dilatation

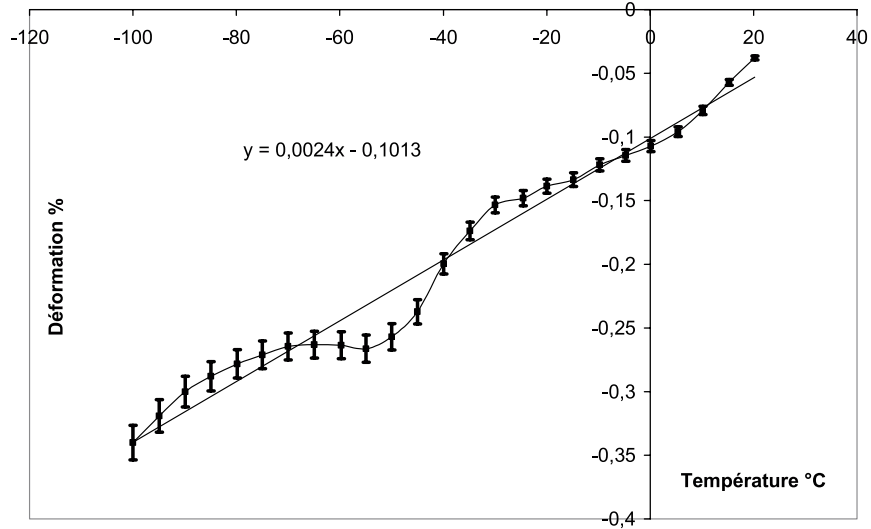

Fig. 4. Déformation mesurée par CCD en fonction de la température.

thermique. L'essai est réalisé de la température ambiante à $-90{ }^{\circ} \mathrm{C}$.

La capture des images se fait tous les $10^{\circ} \mathrm{C}$. Le calcul du coefficient de dilatation est obtenu par la moyenne des déformations de 4 points pris au centre de l'éprouvette. Les résultats choisis pour le calcul sont pris lors de la phase de montée en température (entre $-90{ }^{\circ} \mathrm{C}$ et $23{ }^{\circ} \mathrm{C}$ ). Ce choix permet de s'affranchir des phénomènes d'inertie thermique dans l'enceinte. La figure 4 montre la déformation en fonction de la température lors de la phase de montée en température. La courbe obtenue permet la mesure du coefficient de dilatation moyen $\alpha=24 \times 10^{-6} /{ }^{\circ} \mathrm{C}$.

\subsection{Identification des caractéristiques viscoélastiques}

\subsubsection{Modèle de Maxwell}

Cette phase de l'étude a pour but de déterminer le comportement mécanique du PET/PA/PET. L'objectif de cette partie est de déterminer les coefficients de la loi de comportement à partir d'essais de fluage et de relaxation $[17,18]$. Le polymère utilisé étant fortement nonlinéaire en contrainte, nous avons choisi de le décrire par une loi de comportement de type Maxwell non-linéaire en temps et en contrainte $[14,19,20]$. La loi ainsi identifiée permet de décrire, avec des jeux de coefficients différents, les essais de fluage et de relaxation. Cette phase de l'étude a pour but de déterminer le comportement mécanique du $\mathrm{PET} / \mathrm{PA} / \mathrm{PET}$.

Généralement le modèle « viscoélastique » non-linéaire de Maxwell (pour un polymère visco-élastique) s'écrit comme suit :

$$
\dot{\bar{\varepsilon}}^{c}=A \bar{\sigma}^{m}\left(\bar{\varepsilon}^{c}\right)^{n} T^{p}\left(q t^{q-1}\right)
$$

Dans le cas d'un comportement isotherme l'équation (2) devient :

$$
\dot{\bar{\varepsilon}}=A \bar{\sigma}\left(q t^{q-1}\right)
$$

L'identification des paramètres de la loi est obtenue à partir d'essais de traction uni-axiale. 


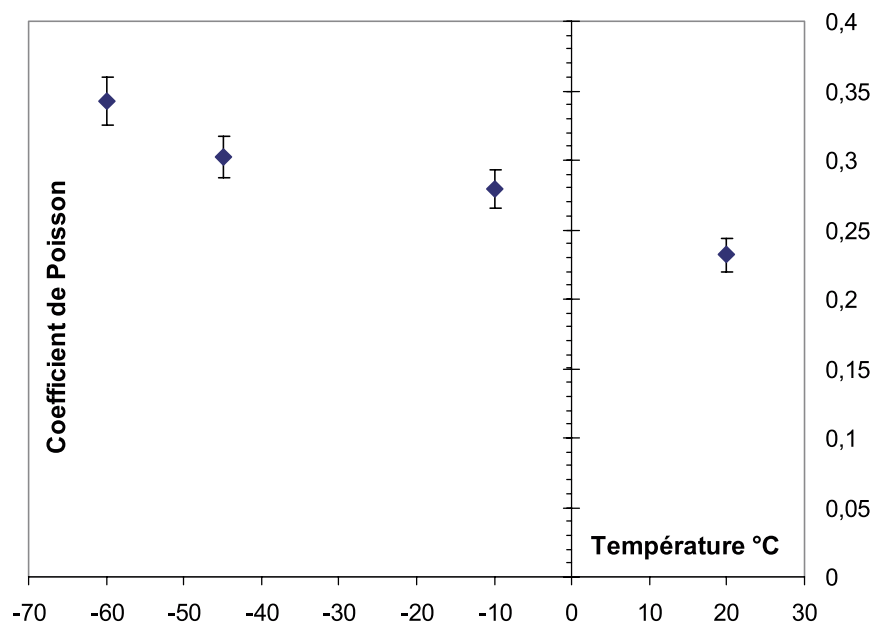

Fig. 5. Évolution du coefficient de Poisson en fonction de la température.

Des tests développés sur le dispositif NIRVANA instrumenté par un système de stéréo-corrélation d'images a permis la mesure du champ de déformations tridimensionnelles. L'utilisation du modèle de Maxwell non-linéaire a permis une bonne représentation du fluage et de la relaxation pour des temps courts. Ce modèle, identifié en fluage donne de bons résultats en fluage mais semble limité dans la description de la relaxation (Fig. 7).

\subsubsection{Modèle spectral non-linéaire (MSNL)}

\subsubsection{Lois constitutives}

Pour décrire le comportement viscoélastique nonlinéaire, nous avons choisi le modèle viscoélastique spectral non-linéaire pour décrire à la fois le fluage et la relaxation [17]. L'écriture tensorielle de ce modèle permet de traiter le caractère multiaxial des sollicitations. La description des phénomènes visqueux en un spectre de temps de relaxation présente l'attrait de se rapprocher sensiblement de la réalité. Enfin, la formulation de ce modèle permet un découplage des paramètres, facilitant le travail d'identification.

Ce modèle se présente donc sous la forme d'un système d'équations différentielles du $1^{\mathrm{er}}$ ordre ayant un nombre de coefficients limité :

$$
\begin{gathered}
\sigma=L:\left(\varepsilon-\varepsilon^{a}\right) \\
\dot{\varepsilon}^{a}=g(\sigma) \cdot \sum_{i} \dot{\xi}_{i}
\end{gathered}
$$

avec

$$
\begin{aligned}
g(\sigma) & =1+\beta\left(\sqrt{\sigma: L_{R}^{-1}: \sigma}\right)^{p} \\
\dot{\xi}_{i} & =\frac{1}{\tau_{i}}\left(\mu_{i} \cdot g(\sigma) \cdot L_{R}^{-1}: \sigma-\xi_{i}\right) \\
\mu_{i} & =\frac{1}{n_{0} \cdot \sqrt{\pi}} \cdot \exp \left(-\left[\frac{i-n_{\mathrm{c}}}{n_{0}}\right]^{2}\right) \text { et } \tau_{i}=e^{n_{i}}
\end{aligned}
$$

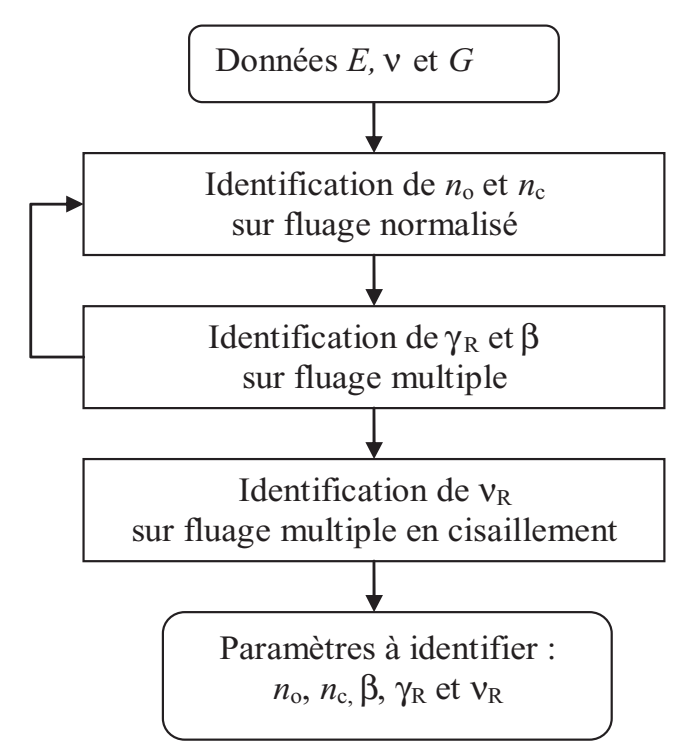

Fig. 6. Principe d'identification du modèle viscoélastique spectral non-linéaire.

La distribution gaussienne est normalisée de sorte que : $\sum_{i=-\infty}^{+\infty} \mu_{i}=1$

$$
L_{R}^{-1}=S_{R}=\left[\begin{array}{ccc}
\gamma_{R} \cdot S_{11} & -\gamma_{R} \cdot S_{11} & 0 \\
-\gamma_{R} \cdot S_{11} & \gamma_{R} \cdot S_{11} & 0 \\
0 & 0 & \gamma_{\mathrm{c}} \cdot S_{66}
\end{array}\right] \begin{aligned}
& \text { avec } \gamma_{\mathrm{c}}=\gamma_{R} \frac{1+v_{R}}{1+v}
\end{aligned}
$$

\subsubsection{Procédure d'identification}

Le modèle de comportement viscoélastique retenu possède au total 8 coefficients à identifier : 2 caractéristiques élastiques $(E$ et $\nu), 2$ coefficients liés à la viscosité $\left(\gamma_{R}\right.$ et $\left.\nu_{R}\right), 2$ à la non-linéarité en contrainte $(\beta$ et $p$ ) et 2 à la répartition spectrale des temps de relaxation $\left(n_{0}\right.$ et $\left.n_{\mathrm{c}}\right)$.

Les caractéristiques élastiques sont directement mesurables sur des courbes $(\sigma-\varepsilon)$ de traction monotone (Sect. 2.2.2). La procédure d'identification peut être schématisée selon le principe décrit dans la figure 6 .

\subsubsection{Résultat}

Nous pouvons constater sur les figures 8 à 10 :

- la prise en compte de la non-linéarité en contrainte,

- un nombre de temps de relaxation élevé pour un nombre de coefficients à identifier réduit,

- un nombre d'essais réduits.

Les résultats sont bien corrélés. Par contre la méthode nécessite une implémentation complexe. 


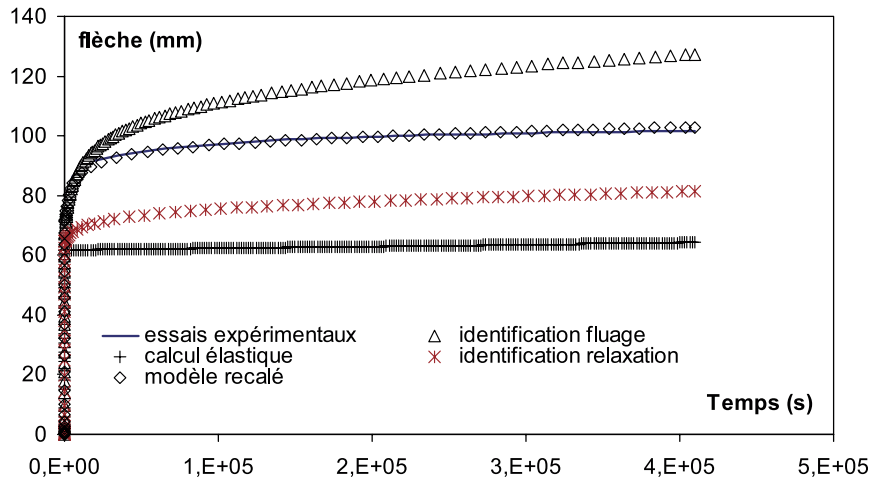

Fig. 7. Comportement en fluage et relaxation d'échantillon avec ruban central à pression constante $(15 \mathrm{hPa})$.

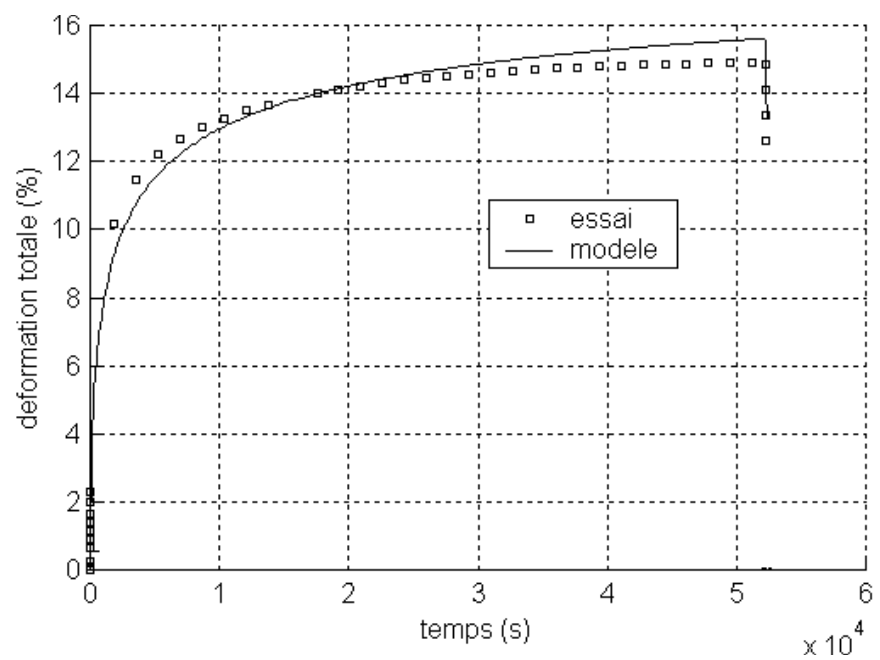

Fig. 8. Déformation en fonction du temps à contrainte constante $(80 \mathrm{MPa})$.

\section{Simulations numériques}

La loi de Maxwell identifiée en fluage est implémentée dans le code éléments finis MSC MARC [][Marc 2003]. Le modèle est un quart de structure maillée en éléments de coque représentant l'échantillon testé sur NIRVANA. Les conditions de pression et de précontrainte en fonction du temps de l'essai de fluage sur NIRVANA sont implémentées directement comme conditions aux limites du calcul. Le couplage du logiciel éléments finis avec un logiciel d'optimisation permet de recaler le modèle. Cette méthode inverse consiste à comparer la flèche au centre de l'échantillon issue de la mesure par stéréo-corrélation avec la même donnée obtenue par le calcul. Le logiciel d'optimisation vient alors modifier via une sous-routine les coefficients de la loi de comportement dans le code éléments finis. Les résultats obtenus montrent que le modèle choisi est représentatif du comportement des sous-ensembles de ballons soumis à des sollicitations complexes (Figs. 8 à 10) et (Figs. 16 et 17).

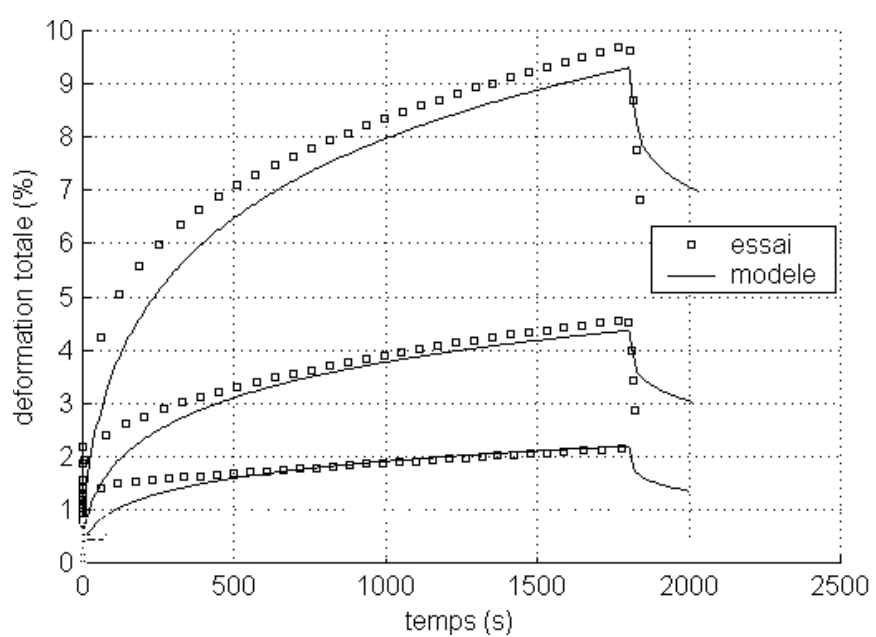

Fig. 9. Déformation en fonction du temps pour différentes charges.

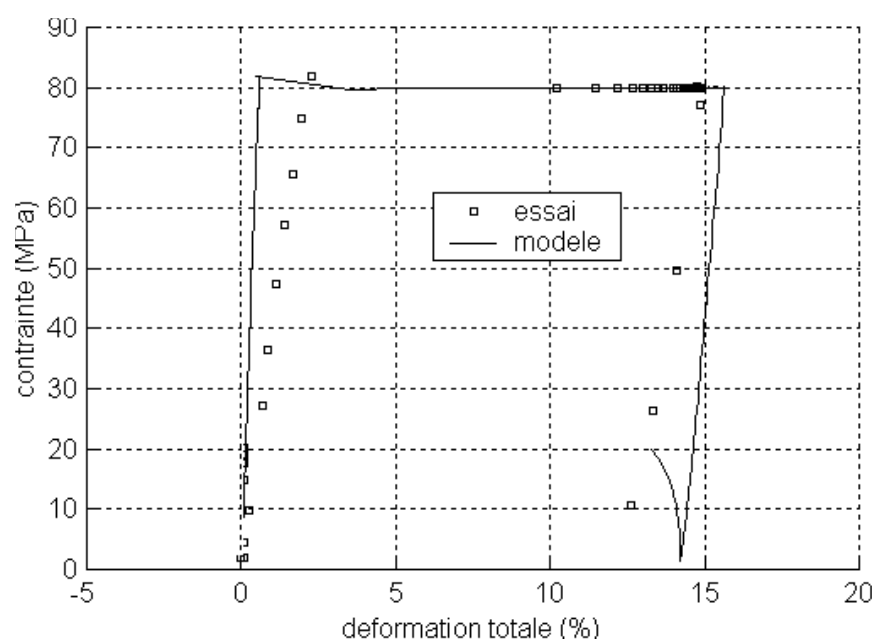

Fig. 10. Contrainte en fonction de la déformation.

\section{Simulations expérimentales}

Ces essais ont pour but de valider les lois de comportement du PET/PA/PET dans des conditions de température et de contrainte représentatives de phases de vol. Ils sont réalisés sur le dispositif expérimental NIRVANA, développé par le CNES et l'ONERA, permettant d'étudier le comportement mécanique de sousensembles de ballons.

\subsection{Dispositif expérimental NIRVANA}

\subsubsection{Présentation}

Il est constitué d'une enceinte à vide réalisée en deux parties séparables dont la géométrie est schématiquement celle de deux demi-cylindres de même diamètre chacun fermé à son extrémité (Fig. 11). L'échantillon étudié est positionné entre les deux demi-cylindres et isole les deux parties de la cuve, il est donc possible d'introduire deux 


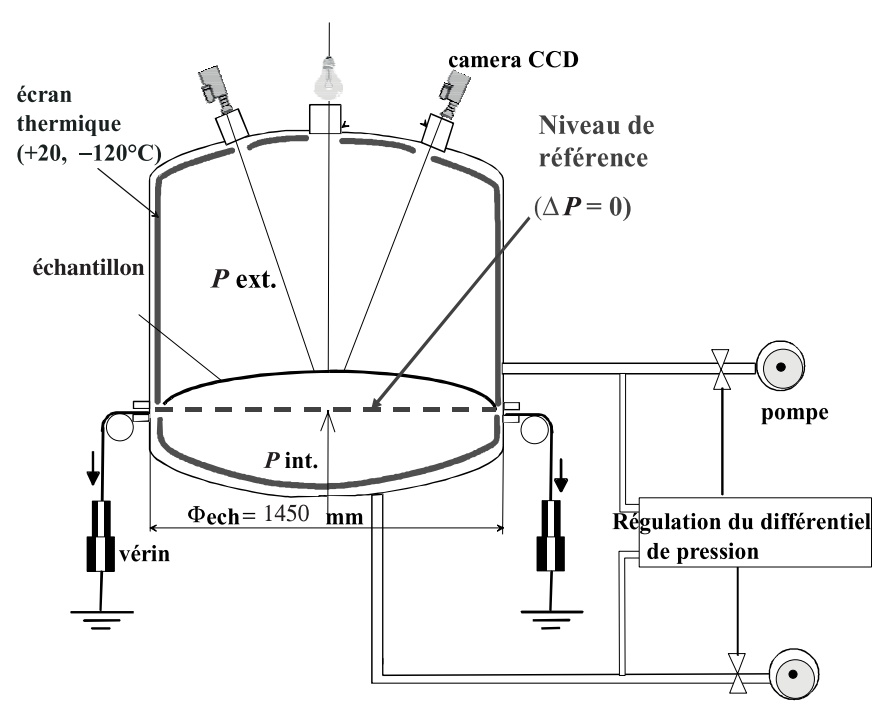

Fig. 11. Dispositif expérimental NIRVANA (vue en coupe).

atmosphères différentes et d'effectuer un différentiel de pression entre les deux volumes créés [9].

Un dispositif constitué de trois vérins pneumatiques asservis et de 150 pinces permet d'accrocher l'échantillon à tester et de lui appliquer une contrainte radiale extérieure.

Une double paroi isolée sous vide scellée sur la périphérie de l'enceinte garantit une isolation thermique par rapport à l'atmosphère extérieure de l'enceinte. Un dispositif interne de circulation d'azote froid dans des écrans thermiques régule la température entre +20 et $-120^{\circ} \mathrm{C}$.

Plusieurs types d'expériences sont réalisables sur le dispositif NIRVANA :

- Étude de la perméabilité à l'hélium de films ou de sous-ensembles de ballons.

- Test d'éclatométrie et de suivi de la déflection de l'échantillon en fonction du différentiel de pression.

- Essais en traction équibiaxiale avec ou sans tension initiale.

- Essais en fluage équibiaxial avec ou sans tension initiale.

\subsubsection{Représentativité du ballon}

Un des rôles du dispositif est de valider, après fabrication, la tenue mécanique de sous-ensembles de ballons dans des conditions proches de la réalité. L'essai effectué doit donc être représentatif d'une phase de vol, en appliquant les conditions de température et de pression. Le système de pré-tension avec vérins permet d'appliquer au film testé la contrainte présente dans la peau du ballon durant le vol [13]. Cette contrainte radiale appliquée en périphérie de l'échantillon simule la présence du reste du ballon (Fig. 12).
On simule ainsi une calotte de ballon dont la précontrainte est donnée par la relation :

$$
\sigma=\frac{\Delta P . R}{2 e} \times 2 \pi \times 1450 \times e
$$

Avec : $\sigma$, la précontrainte à appliquer dans le dispositif NIRVANA, $\Delta P$, la différence de pression entre l'intérieur et l'extérieur du ballon, $R$, le rayon du ballon, $e$, l'épaisseur de l'enveloppe (considérée équivalente pour le ballon complet et l'échantillon à mesurer), $1450 \mathrm{~mm}$ correspond au diamètre de l'échantillon (Fig. 12).

\subsection{3 Éclairage de l'échantillon}

Le problème de l'éclairage vient de la nature réfléchissante et spéculaire du $\mathrm{PET} / \mathrm{PA} / \mathrm{PET}$. Lorsque l'éclairage est dirigé vers l'échantillon, chaque caméra acquiert un reflet spéculaire dans une zone précise.

Ce reflet spéculaire entraîne une surexposition lumineuse dans les zones concernées qui ne permet pas au système de calculer les déplacements et les déformations dans les zones surexposées. La surface totale de l'échantillon mesurée est ainsi réduite de près de $40 \%$.

La solution adoptée pour palier ces problèmes consiste à rendre l'échantillon diffusant. Pour cela, on applique une sous-couche de peinture grise de quelques dixièmes de millimètres d'épaisseur sur l'échantillon rendant sa surface opaque et diffusante. La non homogénéité de cette couche permet en outre de créer un premier dégradé de niveaux de gris, reconnu par le logiciel de traitement d'images.

\subsubsection{Résultats}

Il s'agit de mesurer les déplacements et déformations d'un échantillon, assemblage représentatif d'un fuseau (Fig. 13), soumis à une mise en pression linéaire de 0 à $50 \mathrm{hPa}$.

La figure 14 montre la flèche au centre de l'échantillon en fonction de la différence de pression pour un échantillon avec assemblage central et pré-tension. La figure 15 présente l'évolution de la flèche au centre de l'échantillon en fonction du temps pour ce même échantillon. La figure 16 donne le déplacement vertical ainsi que la déformation normale à l'assemblage de l'ensemble de l'échantillon à un temps donné. On observe bien l'effet de l'assemblage central, d'épaisseur supérieure, qui entraîne une déformation moins importante.

On observe aussi l'influence de l'assemblage sur le déplacement vertical. En effet, l'assemblage plus épais a tendance à créer un champ de déplacement ovale (isodéplacements Fig. 17) plutôt que circulaire.

- Structure à ruban central $\left(T^{\circ}\right.$ ambiante, prétension $=$ $\left.37 \mathrm{~kg} . \mathrm{cm}^{-1}, P_{\text {diff }}=15 \mathrm{hPa}\right)$.

- Calotte $\left(T^{\circ}\right.$ ambiante, prétension $=37 \mathrm{~kg} \cdot \mathrm{cm}^{-1}$, $\left.P_{\text {diff }}=15 \mathrm{hPa}\right)$. 


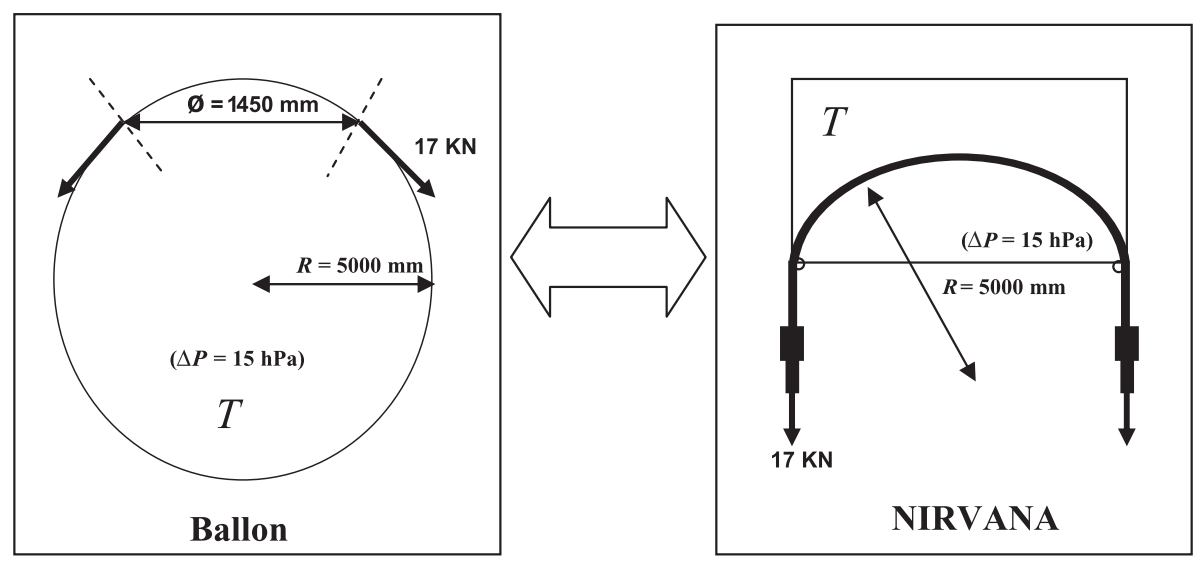

Fig. 12. Représentativité d'un ballon dans le dispositif NIRVANA.
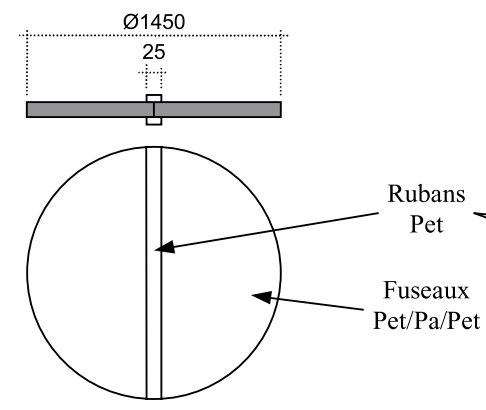

Structure à ruban central

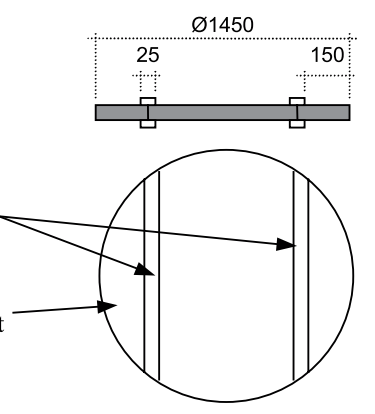

Structure à rubans déportés

Fig. 13. Exemple des deux types de structures testées sur NIRVANA.

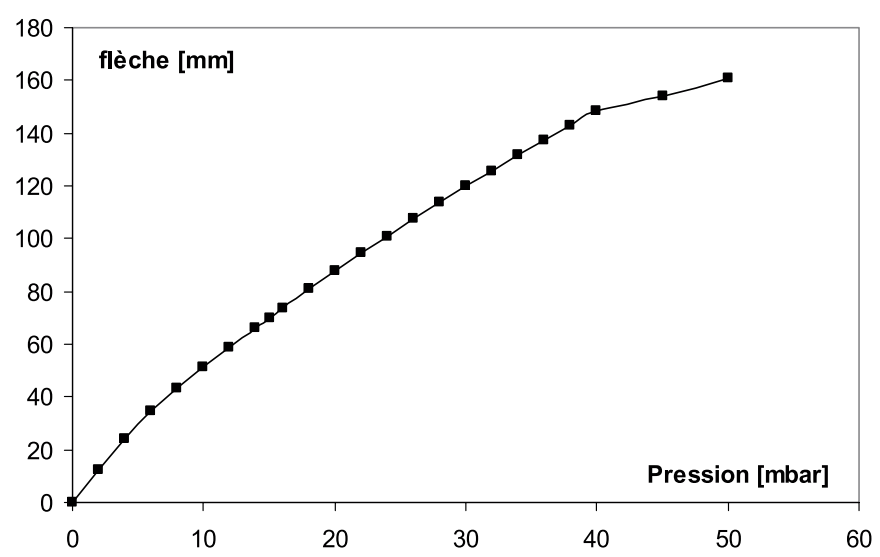

Fig. 14. Évolution de la flèche en fonction de la pression.

\section{Conclusion}

Nous avons étudié le comportement mécanique des ballons pressurisés stratosphériques. L'identification des lois de comportement de films polymères constitutifs des ballons se fait à partir d'essais uniaxiaux en traction monotone, traction de fluage et de relaxation. Ces essais sont réalisés sur une machine de traction équipée d'une caméra CCD. Le dispositif expérimental permet de

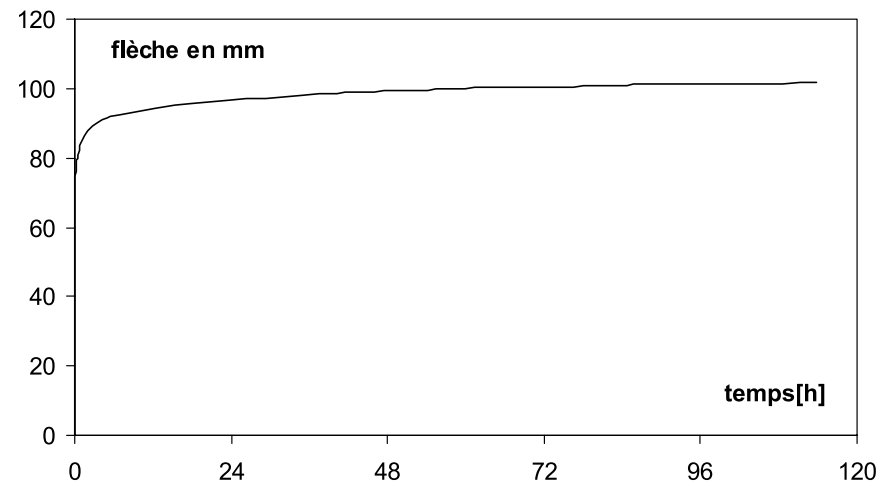

Fig. 15. Évolution de la flèche en fonction du temps.

mesurer les déplacements et d'obtenir les déformations dans le plan de sollicitation de l'éprouvette. On a ainsi accès au module d'Young, au coefficient de Poisson à température ambiante et jusqu'à $-60{ }^{\circ} \mathrm{C}$, ainsi qu'au coefficient de dilatation du film complexe de PET/PA/PET. Des procédures d'essais adaptées à l'identification de matériaux souples et transparents ont été ainsi mises en place.

La partie expérimentale destinée à valider les lois de comportement est réalisée à partir du dispositif expérimental NIRVANA équipé d'un système de mesure par stéréo-corrélation. Ce procédé d'extensométrie sans contact autorise la mesure des déplacements 3D de grands sous-ensembles de ballons constitués de matériaux souples et transparents. Les déformations calculées permettent de constituer une base de données expérimentale utilisée pour la connaissance du comportement des ballons ainsi que pour la validation des lois de comportement.

Une simulation par éléments finis des essais réalisés sur le dispositif expérimental permet, par comparaison des champs de déplacements, de valider les lois de comportement. 


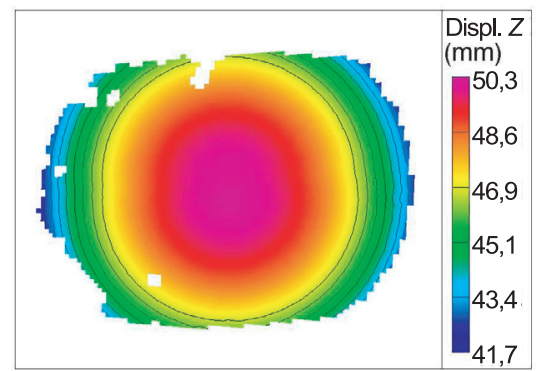

(a) Déplacement vertical mesuré

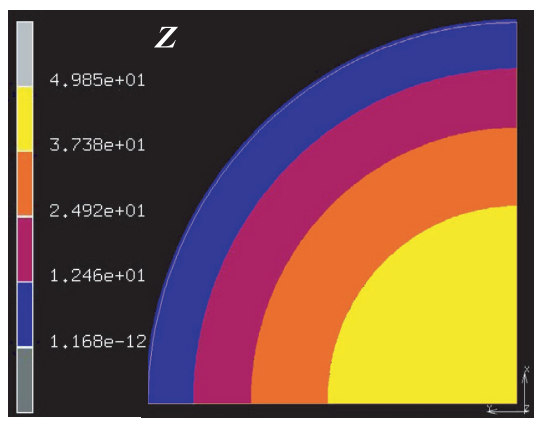

(c) Déplacement vertical numérique

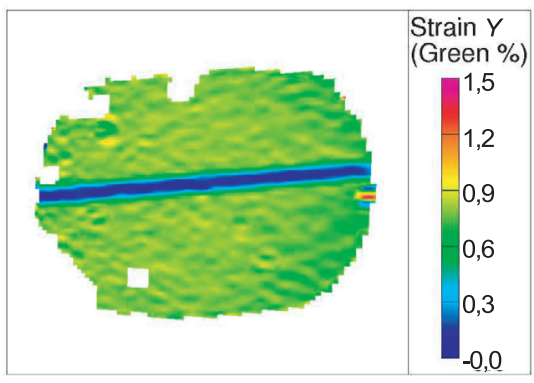

(b) Déformation mesurée suivant $Y$

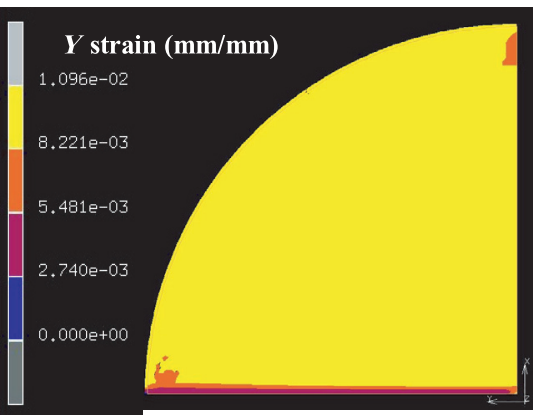

(d) Déformation numérique suivant $Y$

Fig. 16. Déplacements et déformations pour une structure avec ruban central.

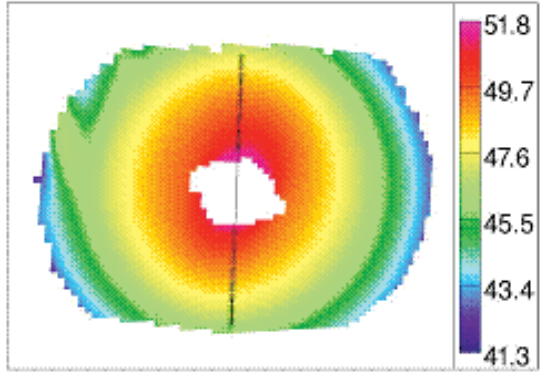

(a) Déplacement vertical mesuré

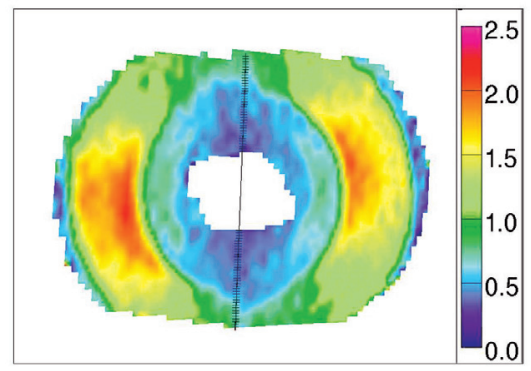

(c) Déformation mesurée suivant $Y$

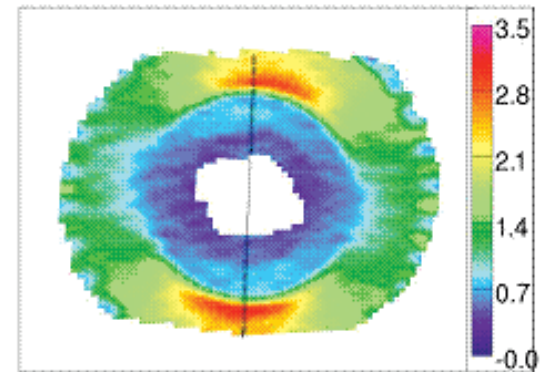

(b) Déformation mesurée suivant $X$

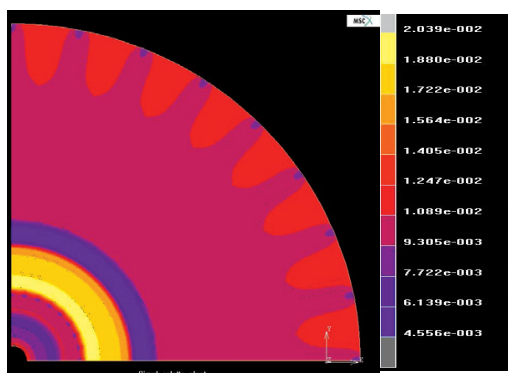

(d) Déformation numérique suivant $X$

Fig. 17. Déplacements et déformations pour une calotte. 


\section{Références}

[1] J.J. Aklonis, Introduction to Polymer Viscoelasticity, Wiley, 1995

[2] P. Cocquerez, P. Guigue, R. Guilbon, T. Phulpin, M. Durand, J.P. Lefèvre, M. Eymard, M. Lafourcade, Test flight of CNES stratospheric super-pressure balloons in experimental arctic campaigns 2000-2001, Objectives and results, Proceedings of 15th ESA Symp., on European Rocket and Balloon Prog., Biarritz, France, May 2001, pp. 597-601

[3] P. Guigue, J.M. Siguier, Engineering of stratospheric balloon materials: selection procedure, experimental characterization and aging techniques, Proceedings of 15th ESA Symp., on European Rocket and Balloon Prog., 2001, pp. $547-552$

[4] M. Karama, S. Mistou, B. Lorrain, Mesures de déformations en 3D basées sur les techniques de photogrammétrie et d'analyse des distributions de niveaux de gris, Revue Instrumentation et Systèmes 207 (2001) $32-35$

[5] D. Garcia, Mesure de formes et de champs de déplacements tridimensionnels par stéréo-corrélation d'images, Thèse de l'École des mines d'Albi, 2001

[6] M.A. Sutton, S.R. McNeill, J.D. Helm, Y.J. Chao, Advances in two-dimensional and three-dimensional computer vision, Photomechanics, Topics Applied Physics 77 (2000) 323-372

[7] S. Mistou, M. Karama, O. Dalverny, J.M. Siguier, P. Guigue, Mesure 3D sans contact des déplacements et déformations sur des films plastiques transparents par stéréo-corrélation, Mécanique \& Industries 4 (2003) 637-643

[8] P. Vialettes, J.M. Siguier, P. Guigue, S. Mistou, O. Dalverny, M. Karama, F. Petitjean, Étude par stéréocorrélation des champs de déplacement et de déformation de sous-ensembles de ballons stratosphériques pressurisés, Revue Instrumentation, Mesure, Métrologie, I2M, 200505 (à paraître)

[9] J.M. Siguier, P. Vialettes, S. Granier, P. Guigue, The use of Nirvana facility for balloon characterization: Strain and tightness measurements of zero pressure and superpressure balloon subsystems, Proceedings of 16th ESA Symp., on European Rocket and Balloon Prog., Sankt Gallen, Switzerland, June 2003, pp. 207-213

[10] J.-M. Siguier, P. Guigue, M. Karama, S. Mistou, O. Dalverny, S. Granier, Numerical and experimental simulation of the mechanical behavior of super-pressure balloon subsystems, 34th COSPAR, Houston, USA, 10-19 October, 2002

[11] M. Grédiac, The use of full-field measurement methods in composite material characterization: interest and limitations, Composites Part A: Applied Science and Manufacturing 35(7-8) (2004) 751-761

[12] J.-J. Orteu, V. Garric, M. Devy, Camera calibration for $3 \mathrm{D}$ reconstruction: application to the measure of $3 \mathrm{D}$ deformations on sheet metal parts, European Symposium on Lasers, Optics and vision in manufacturing, Munich, Allemagne, June, 1997

[13] P. Vialettes, J.M. Siguier, P. Guigue, S. Mistou, O. Dalverny, M. Karama, F. Petitjean, Identification du comportement mécanique des ballons pressurisés stratospheriques, Revue des composites et materiaux avancés, septembre 2004, à paraître

[14] P. Vialettes, J.M. Siguier, P. Guigue, O. Dalverny, S. Mistou, F. Petitjean, M. Karama, Modélisation du comportement non-linéaire de films minces constitutifs des ballons pressurisés stratosphériques, $16^{\text {e }}$ Congrès Français de Mécanique Nice, 1-5 septembre, 2003

[15] A. Schieffer, Modélisation multiéchelle du comportement mécanique des composites à matrice organique et effets $\mathrm{du}$ vieillissement thermique, Thèse de doctorat, Université de Technologie de Troyes, 2003

[16] Ch. Remy-Petipas, Analyse et prévision du comportement à long terme des composites fibres de carbone/matrice organique, Thèse de doctorat, No d'ordre 801, Université de Franche-Comté, 2000

[17] J.F. Maire, O. Lesné, C. Petipas, une nouvelle approche de la modélisation du comportement visqueux des composites organiques, $10^{\mathrm{e}}$ journées nationales sur les composites, JNC, 1996

[18] J.F. Maire, Études théorique et expérimentale du comportement de matériaux composites en contraintes planes, Thèse de doctorat, $\mathrm{N}^{\mathrm{o}}$ d'ordre 282 , Université de Franche-Comté, 1992

[19] A.S. Nowick, B.S. Berry, Anelastic Relaxation in crystalline Solids Academic Press, 1972

[20] J. Lemaitre, J.L. Chaboche, Mécanique des matériaux solides, Dunod, France, 1985

[21] M. Karama, S. Mistou, P. Peres, G. Gohorianu, Mesure de déformations sans contact sur des composites carbone/carbone à architecture 3D, Troisième colloque francophone sur les méthodes et techniques optiques pour l'industrie, Saint Aubin de Médoc, 18-22 novembre, 2002 